\title{
Scanning Probe Microscopy in TEM : an In-situ Approach for Nano-scale Property Measurements
}

\author{
Zhong Lin (ZL) Wang*
}

Center for Nanoscience and Nanotechnology, and School of Materials Science and Engineering, Georgia Institute of Technology, Atlanta, GA 30332-0245.

* e-mail: zhong.wang@mse.gatech.edu

Property characterization of nanomaterials is challenged by the small size of the structure because of the difficulties in manipulation. Here we demonstrate a novel approach that allows a direct measurement of the mechanical properties, the electron field emission and the ballistic quantum conductance in individual nanotubes and nanowires by an conjunction operation of scanning probe microscopy in transmission electron microscopy (TEM). The technique is powerful in a way that it can directly correlate the atomic-scale microstructure of the carbon nanotube with its physical properties, providing a one-to-one correspondence in structure-property characterization.

Bending modulus of a carbon nanotube: To carry out the property measurement of a nanotube, a specimen holder for an TEM was built for applying a voltage across a nanotube and its counter electrode [1]. Static and dynamic properties of the nanotubes can be obtained by applying controllable static and alternating electric fields. To measure the bending modulus of a carbon nanotube, an oscillating voltage is applied on the nanotube with ability to tune the frequency of the applied voltage. Resonance can be induced in carbon nanotubes by tuning the frequency (Figure 1), from which the bending modulus can be derived. For nanotubes produced by arc-discharge, which are believed to be defect-free, the bending modulus is as high as $1.2 \mathrm{TPa}$ (as strong as diamond) for nanotubes with diameters smaller than $8 \mathrm{~nm}$, and it drops to as low as $0.2 \mathrm{TPa}$ for those with diameters larger than $30 \mathrm{~nm}$ [1]. Nanotubes produced by catalystassisted pyrolysis containing a high density of point defects have bending modulus of 7 times smaller than that of a defect-free nanotube $[2]$.

Electron field emission from individual carbon nanotubes: For carbon nanotube emitters, most of the electrons are emitted from the tips of the tubes, and it is the local work function that matters to the properties of the tube field emission. We present a novel approach for measuring the tip work functions of individual carbon nanotubes $[3,4]$. Our results indicate that the tip work function show no significant dependence on the diameter of the nanotubes in the range of $1455 \mathrm{~nm}$. Majority of the nanotubes $(\sim 75 \%)$ have a work function of 4.6-4.8 $\mathrm{V}$ at the tips, which is 0.2-0.4 $\mathrm{V}$ lower than that of carbon. A small fraction of the tubes $(\sim 25 \%)$ have a work function of $\sim 5.6 \mathrm{~V}$, about $0.6 \mathrm{~V}$ higher than that of carbon. Field emission induced structural damage has also been observed and the results explain the current fluctuation in field emission [5].

Ballistic conductance in carbon nanotubes: The conductance of a carbon nanotube was measured as a function of the depth with which the tube was inserted into the mercury (Figure 3) [6]. Surprisingly, the nanotube displays quantum conductance No heat dissipation was observed in the nanotube. This is the ballistic conductance, and it is believed to be a result of single graphite layer conductance. 
[1] P. Poncharal, Z.L. Wang, D. Ugarte, and W.A. de Heer, Science 283 (1999) 1516;

Z.L. Wang, P. Poncharal and W.A. De Heer, Pure Appl. Chem., 72, Nos. 1-2 (2000) 209;

Z.L. Wang, P. Poncharal, and W.A. De Heer, J. Physics \& Chemistry of Solids, 61 (2000) 1025.

[2] R.P. Gao, Z.L. Wang, Z.G. Bai, W. de Heer, L. Dai and M. Gao, Phys. Rev. Letts., 85 (2000) 622.

[3] Z.L. Wang, P. Poncharal, and W.A. De Heer, Microscopy and Microanalysis, 6 (2000) 224.

[4] R.P. Gao, Z.W. Pan and Z. L. Wang Appl. Phys. Letts., , 78 (2001) 1757.

[5] Z.L. Wang, R.P. Gao, W. de Heer and P. Poncharal, Appl. Phys. Letts., in press (2002).

[6] S. Frank, P. Poncharal, Z.L. Wang, and W.A. de Heer,Science 280 (1998) 1744.

[7] Thanks to the support from the NSF grant DMR-9733160 and Georgia Tech. This work was collaboratively carried out with P. Poncharal, W. de Heer and R.P. Gao.
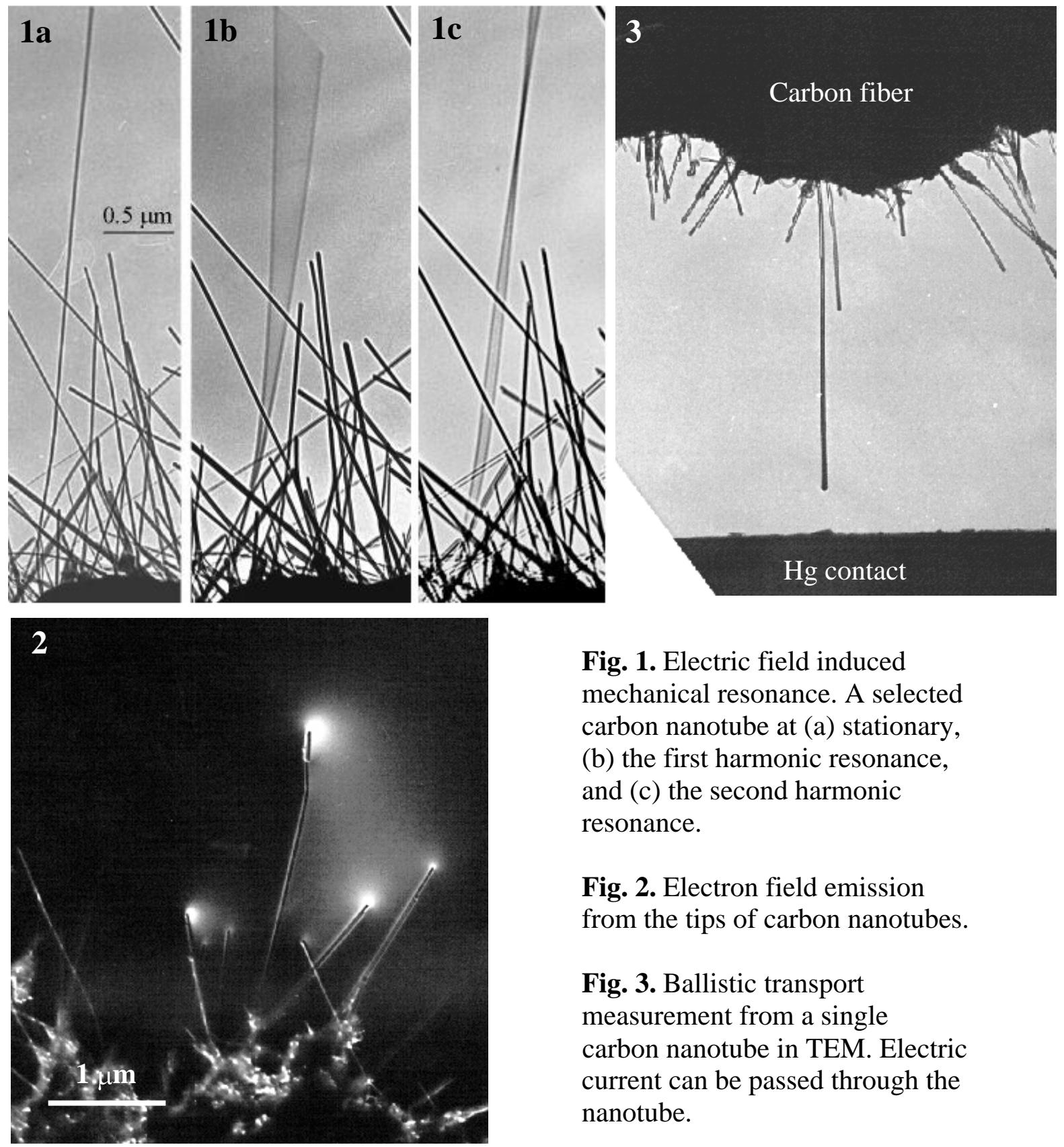

Fig. 1. Electric field induced mechanical resonance. A selected carbon nanotube at (a) stationary, (b) the first harmonic resonance, and (c) the second harmonic resonance.

Fig. 2. Electron field emission from the tips of carbon nanotubes.

Fig. 3. Ballistic transport measurement from a single carbon nanotube in TEM. Electric current can be passed through the nanotube. 